\title{
ESTIMATION OF THE COEFFICIENT OF VARIATION OF THE EXCESS OF LOSS BY MEANS OF JENSEN'S INEQUALITY FOR CONVEX FUNCTIONS. THE CONNECTION WITH THE THEORY OF LIFE TIMES
}

\author{
J. van Kimnen and C. J. GroenenberG \\ Amsterdam
}

This note deals with a way to determine upper and lower bounds for the coefficient of variation of the total claim costs in a year in excess of a certain limit value. In many a publication attention has been paid to the question of calculating, in addition to the mean of the excess costs, the variance of these costs. In this connection we only mention here the study of Vajda: Mirimum Variance Reinsurance, ASTIN-Bulletin, September 1962. It is evident that in studying such problems of "statistics of large claims" the coefficient of variation, defined as the ratio of the standard deviation and the mean, may be a useful tool. If we exclude special "dangerous" claim distributions and also distributions with a very "irregular" tail, it appears possible to derive bounds for this coefficient of variation and to indicate its asymptotic behaviour. Hereby good use can be made of Jensen's inequality for convex functions. Jensen's inequality has frequently been applied to problems in the field of life insurance mathematics, but as far as the authors of this note know, not to the question mentioned here.

Besides, it may have some interest to point out a connection between the question of estimating the excess of total claim costs and the theory of life times. We may translate "the limit value" as "the age already reached" and "the mean of the excess of loss" as "the expectation of life". The estimation of the coefficient of variation of the life time now leads to some well-known biometric formulae on life times and at the same time to an interesting observation as regards human life tables.

The following remarks may be considered as to belong to the field of statistics of large claims. A number of studies in recent 
years have been devoted to this topic by ASTIN-members. In a certain sense this note continues this line and I am glad to have the opportunity to inform you on the results of our small investigations. As the complete proofs of the inequalities and estimates are rather lengthy they will only be sketched here.

I. Definition of the coefficient of variation of the eXCESS OF LOSS

Let $x$ be the total costs in a year, $F(x)$ the corresponding distribution function and $X$ the-non random-limit value. We are interested in the properties of the distribution of $x-X$ with the condition $x \geq X$. The $k^{\text {th }}$ moment of $x-X$ is given by:

$$
M_{k}(X)=\frac{\mathrm{I}}{\mathrm{I}-F(X)} \int_{x}^{\infty}(x-X)^{k} d F(x)
$$

The-square of the-coefficient of variation of $x-X$ is defined by:

$$
\text { C.V. }{ }^{2}(X)=\frac{M_{2}(X)}{M_{1}^{2}(X)}-\mathbf{I}
$$

The reinsurer has also to reckon with the probability of no excess of loss, i.e.: $x<X$. We define further:

$$
\tilde{M}_{k}(X)=\int_{x}^{\infty}(x-X)^{k} d F(x)
$$

Obviously

$$
\tilde{M}_{k}(X)=\tilde{M}_{0}(X) M_{k}(X)
$$

Hence he will be interested in the behaviour of:

$$
\begin{aligned}
\mathrm{C} . \mathrm{V} .{ }^{2}(X) & =\frac{\tilde{M}_{2}(X)}{\tilde{M}_{1}^{2}(X)}-\mathrm{I}=\frac{\mathrm{I}}{\tilde{M}_{0}(X)} \frac{M_{2}(X)}{M_{1}^{2}(X)}-\mathrm{I} \\
& =\frac{\mathrm{I}}{\tilde{M}_{0}(X)}\left(\mathrm{C} . V .{ }^{2}(X)+\mathrm{I}\right)-\mathrm{I}
\end{aligned}
$$

We obtain already the trivial outcome:

$$
\widetilde{C . V .}{ }^{2}(X)>\frac{\mathrm{I}}{\tilde{M}_{0}(X)}-\mathrm{I}=\frac{F(X)}{\mathrm{I}-F(X)}
$$

and

$$
\lim _{x \rightarrow \infty} \tilde{\operatorname{C.V}}{ }^{2}(X)=+\infty
$$


We propose to describe some properties of $\mathrm{C} . \mathrm{V} .{ }^{2}(X)$ in relation to $M_{1}(X)$. Afterwards these properties can be translated in terms of $\widetilde{C . V} .^{2}(X)$ and $\tilde{M}_{1}(X)$ by means of the formulas just given.

2. VARIOUS EXPRESSIONS FOR THE MOMENTS $M_{k}(X)$.

With the view of deriving formula's for C.V. ${ }^{2}(X)$ which can be used in connection with the inequality of Jensen we firstly mention some formulas and relations for $M_{k}(X)$ and the intensity function

$$
\mu(x)=\frac{I}{I-F(x)} \frac{d F(x)}{d x} .
$$

We expressly assume that the moments exist for at least $k=2$ and that the common rules for partial integration and differentiation of an integral to a parameter are applicable. Especially we shall suppose:

$$
\lim _{x \rightarrow \infty}(x-X)^{k} \exp \left[-\int_{x}^{x} \mu(\xi) d \xi\right]=0
$$

Introducing the intensity function $\mu(x)$ we can write:

$$
\begin{gathered}
M_{k}(X)=\int_{x}^{\infty}(x-X)^{k} \frac{\mathrm{I}}{\mathrm{I}-F(x)} \frac{d F(x)}{d x} \frac{\mathrm{I}-F(x)}{\mathrm{I}-F(X)} d x \\
=\int_{\boldsymbol{x}}^{\infty}(x-X)^{k} \mu(x) \exp \left[-\int_{x}^{x} \mu(\xi) d \xi\right] d x
\end{gathered}
$$

Partial integration of (2.I) leads to:

$$
M_{k}(X)=k \int_{x}^{\infty}(x-X)^{k-1} \exp \left[-\int_{x}^{x} \mu(\xi) d \xi\right] d x
$$

Differentiation of (2.2) to the parameter $X$ and taking into account that $\mu(x) \geqslant 0$, gives:

$$
\left\{\begin{array}{l}
\frac{d M_{k}(X)}{d X}=\mu(X) M_{k}(X)-k M_{k-1}(X) \\
\frac{d M_{k}(X)}{d X} \geqslant-k M_{k-1}(X)
\end{array}\right.
$$

The formal quadrature of $(2.3)$ leads to:

$$
M_{k}(X)=k \int_{x}^{\infty} M_{k-1}(x) \exp \left[-\int_{x}^{x} \mu(\xi) d \xi\right] d x
$$


Finally, the substitution of $\mu(x)$ from (2.3) into (2.4) for $k-\mathrm{I}$ results in:

$$
\begin{aligned}
& M_{k}(X)=k \int_{x}^{\infty} M_{k-1}(x) \exp \left[-\int_{x}^{x}\left\{\frac{\mathrm{I}}{M_{k-1}(\xi)} \frac{d M_{k-1}(\xi)}{d \xi}+\right.\right. \\
& \left.\left.+(k-\mathrm{I}) \frac{M_{k-2}(\xi)}{M_{k-1}(\xi)}\right\} d \xi\right] d x \\
& =k M_{k-1}(X) \int_{x}^{\infty} \exp \left[-(k-\mathrm{I}) \int_{x}^{x} \frac{M_{k-2}(\xi)}{M_{k-1}(\xi)} d \xi\right] d x
\end{aligned}
$$

3. EXPRESSiONS FOR THE COEFFICIENT OF VARIATION (C.V. $(X)$

The differential equation for $M_{k}(X),(2.3)$ leads to a differential equation for C.V. ${ }^{2}(X)$. We find:

$$
\frac{d \mathrm{C} . \mathrm{V} .{ }^{2}(X)}{d X}=\mathrm{C} . \mathrm{V} .{ }^{2}(X)\left(\frac{2}{M_{1}(X)}-\mu(X)\right)-\mu(X)
$$

Furthermore, if we replace $\mu(X)$ by $\frac{\mathrm{I}}{M_{1}(X)}\left(\mathrm{I}+\frac{d M_{1}(X)}{d X}\right)$ we obtain:

$$
\begin{aligned}
\frac{d \mathrm{C} \cdot \mathrm{V} \cdot{ }^{2}(X)}{d X} & =\mathrm{C} \cdot \mathrm{V} \cdot{ }^{2}(X) \frac{\mathrm{I}}{M_{1}(X)}\left(\mathrm{I}-\frac{d M_{1}(X)}{d X}\right)- \\
& -\frac{\mathrm{I}}{M_{1}(X)}\left(\mathrm{I}+\frac{d M_{1}(X)}{d X}\right)
\end{aligned}
$$

We can write down explicit solutions at once by means of the formulas for $M_{k}(X), k=\mathrm{I}, 2$, given in the forgoing section. In particular we have:

$$
\begin{aligned}
M_{1}(X) & =\int_{x}^{\infty} \exp \left[-\int_{x}^{x} \mu(\xi) d \xi\right] d x \\
M_{2}(X) & =2 \int_{x}^{\infty}(x-X) \exp \left[-\int_{x}^{x} \mu(\xi) d \xi\right] d x \\
& =2 \int_{x}^{\infty} M_{1}(x) \exp \left[-\int_{x}^{x} \mu(\xi) d \xi\right] d x \\
& =2 M_{1}(X) \int_{x}^{\infty} \exp \left[-\int_{x}^{x} \frac{d \xi}{M_{1}(\xi)}\right] d x
\end{aligned}
$$


and in consequence:

$$
\begin{gathered}
\text { C.V. }{ }^{2}(X)=\frac{M_{2}(X)}{M_{1}^{2}(X)}-\mathrm{I} \\
=\frac{2}{M_{1}(X)} \frac{\int_{x}^{\infty}(x-X) \exp \left[-\int_{x}^{x} \mu(\xi) d \xi\right] d x}{\int_{x}^{\infty} \exp \left[-\int_{x}^{x} \mu(\xi) d \xi\right] d x}-\mathrm{I} \\
=\frac{2 \int_{x}^{\infty} \frac{M_{1}(x)}{M_{1}(X)} \exp \left[-\int_{x}^{x} \mu(\xi) d \xi\right] d x}{\int_{x}^{\infty} \exp \left[-\int_{x}^{x} \mu(\xi) d \xi\right] d x}-\mathrm{I} \\
=\frac{\int_{x}^{\infty} \exp \left(\int_{x}^{x}\left(\mu(\xi)-\frac{\mathrm{I}}{M_{1}(\xi)}\right) d \xi\right] \exp \left[-\int_{x}^{x} \mu(\xi) d \xi\right] d x}{\int_{x}^{\infty} \exp \left[-\int_{x}^{x} \mu(\xi) d \xi\right] d x}-\mathrm{I}
\end{gathered}
$$

The formula (3.4) shows already that:

$$
\text { C.V. }{ }^{2}(X)(\$) \text { I if } \frac{d M_{1}(X)}{d X}(\$) \circ, X \geq X_{0}
$$

Applying this result to (3.3) we obtain the inequality:

$$
\frac{1}{2} M_{1}(X) \leq \frac{\int_{x}^{\infty}(x-X) \exp \left[-\int_{x}^{x} \mu(\xi) d \xi\right] d x}{\int_{x}^{\infty} \exp \left[-\int_{x}^{x} \mu(\xi) d \xi\right] d x} \leq M_{1}(X)
$$

if

$$
\begin{gathered}
M_{1}^{\prime}(X) \leq 0, X \geq X_{0} \\
M_{1}(X) \leq \frac{\int_{X}^{\infty}(x-X) \exp \left[-\int_{x}^{x} \mu(\xi) d \xi\right] d x}{\int_{x}^{\infty} \exp \left[-\int_{x}^{x} \mu(\xi) d \xi\right] d x},
\end{gathered}
$$

if

$$
M_{1}^{\prime}(X) \geq 0, X \geq X_{0} \text {. }
$$


(remark: if we interpret $x-X$ as the time still to live after reaching age $X, \mu(x)$ as the intensity of dying and in consequence $M_{1}(X)$ as the expectation of life, we get an extension of a well-known biometric theorem on life times).

$$
\text { 4. BOUNDS FOR C.V. }{ }^{2}(X) \text { AND } \lim _{X \rightarrow \infty} \operatorname{C.V} .^{2}(X)
$$

What follows now is a direct application of Jenson's inequality. Jensen's inequality reads as:

$$
\begin{aligned}
& \frac{\int_{x}^{\infty} \varphi(x) f(x) d x}{\int_{x}^{\infty} f(x) d x} \gtrless\left(\frac{\int_{x}^{\infty}(x-X) f(x) d x}{\int_{x}^{\infty} f(x) d x}\right) \\
& \text { if } \frac{d^{2} \varphi(x)}{d x^{2}}(\gtreqless) 0, x>X, f(x)>0
\end{aligned}
$$

We translate $\varphi(x)$ and $f(x)$ into:

$$
\begin{gathered}
f(x) \stackrel{\text { def }}{=} \exp \left[-\int_{x}^{x} \mu(\xi) d \xi\right] \\
\varphi(x) \stackrel{\text { def }}{=} \exp \left[\int_{x}^{x}\left(\mu(\xi)-\frac{I}{M_{1}(\xi)}\right) d \xi\right]
\end{gathered}
$$

From (2.3) for $k=\mathrm{I}$ it follows that:

$$
\begin{aligned}
\exp \left[\int_{x}^{x}\left(\mu(\xi)-\frac{\mathrm{I}}{M_{1}(\xi)}\right) d \xi\right] & =\frac{M_{1}(x)}{M_{1}(X)} \text {, hence } \\
& \frac{d^{2} \varphi(x)}{d x^{2}}=\frac{\mathrm{I}}{M_{1}(X)} \cdot M_{1}^{\prime \prime}(x)
\end{aligned}
$$

Further if we define:

$$
\bar{x}-X \stackrel{\operatorname{def}}{=} \frac{\int_{x}^{\infty}(x-X) f(x) d x}{\int_{x}^{\infty} f(x) d x},
$$

we see from (3.3) that:

$$
\bar{x}-X=\frac{M_{1}(X)}{2}\left(\text { C.V. }{ }^{2}(X)+\mathrm{I}\right)
$$


We now propose to study the most important cases, a.

$$
M_{1}^{\prime}(X) \leq 0, \quad M_{1}^{\prime \prime}(X) \geq 0, \quad X \geq X_{0}
$$

These conditions imply: $\quad \mu^{\prime}(x) \geq 0$

Application of Jensen's inequality in conjunction with (3.5), (3.6) and (3.7) yields the inequality:

$$
2 \exp \left[\int_{x}^{\bar{x}}\left(\mu(\xi)-\frac{\mathrm{I}}{M_{1}(\xi)}\right) d \xi\right]-\mathrm{I} \leq \mathrm{C} \cdot \mathrm{V} \cdot{ }^{2}(X) \leq \mathrm{I}
$$

with $\bar{x}$ given by (4.4).

To obtain a useful lower bound for C.V.a $(X)$ we have to estimate the integral:

$$
\int_{x}^{\bar{x}}\left(\mu(\xi)-\frac{I}{M_{1}(\xi)}\right) d \xi=\int_{x}^{\bar{x}} \frac{d \log M_{1}(\xi)}{d \xi} d \xi
$$

If $\frac{d^{2} \log M_{1}(x)}{d x^{2}} \geq 0$ we find in relation with (4.4):

$\left|\int_{x}^{\bar{z}}\left(\mu(\xi)-\frac{\mathrm{I}}{M_{1}(\xi)}\right) d \xi\right| \leq M_{1}(X) \frac{\mathrm{I}}{M_{1}(X)}\left|M_{1}^{\prime}(X)\right|=\left|M_{1}^{\prime}(X)\right|$

Hence we finally obtain:

$$
2 \exp \left[M_{1}^{\prime}(X)\right]-\mathrm{I} \leq \mathrm{C} \cdot \mathrm{V}^{2}(X) \leq \mathrm{I}
$$

Furthermore if:

$$
\lim _{X \rightarrow \infty} M_{1}^{\prime}(X)=0
$$

holds, we get:

$$
\lim _{X \rightarrow \infty} \mathrm{C} . V .^{2}(X)=\mathrm{I}
$$

(remark: It is tempting to relate these results with observations on human life tables. For recent life tables it is easily verified that we have for the-graduated-function of the complete expectation of life:

$$
\frac{d \dot{e}(x)}{d x} \leq 0, \frac{d^{2} \dot{e}(x)}{d x^{2}} \geq 0 \text {, but not: } \frac{d^{2} \log \dot{e}(x)}{d x^{2}} \geq 0
$$


Therefore the analysis just described for obtaining interval estimates for the coefficient of variation is too rough and has to be modified somewhat. From (3.4) we may conclude:

$$
\bar{x}<x+\stackrel{e}{e}(x)
$$

and in consequence we get:

$$
2 \frac{\grave{e}(x+\check{e}(x))}{\grave{e}(x)} \leq \mathrm{C} . \mathrm{V} .^{2}(x) \leq \mathrm{I}
$$

As the abundant statistics with regard to life expectations clearly show that $\frac{\grave{e}(x+\check{e}(x)}{\check{e}(x)}$ is an increasing function which tends to one, we again may conclude that: $\lim _{x \rightarrow \infty} \mathrm{C} . \mathrm{V} .{ }^{2}(x)=\mathbf{r}$.

Therefore the coefficient of variation increases with $x$ and tends to one. The estimation procedure of the time still to live can, in a certain sense, said to be inconsistent).

The other cases can be treated along the same lines:

b.

$$
M_{1}^{\prime}(X) \leq 0, \quad M_{1}^{\prime \prime}(X) \geq 0, \quad X \geq X_{0}
$$

We find:

$$
0 \leq \mathrm{C} \cdot V \cdot{ }^{2}(X) \leq 2 \exp \left[\int_{x}^{\bar{x}}\left(\mu(\xi)-\frac{\mathrm{I}}{M_{1}(\xi)}\right) d \xi\right]-\mathrm{I}
$$

with:

$$
\bar{x}-X \geq \frac{1}{2} M_{1}(X)
$$

As now $\frac{d^{2} \log M_{1}(X)}{d X^{2}} \leq 0$, we obtain:

$$
0 \leq \mathrm{C} \cdot \mathrm{V}^{2}(X) \leq 2 \exp \left[\frac{1}{2} M_{1}^{\prime}(X)\right]-\mathrm{I}
$$

From the differential equation (3.2) we may conclude that in the case:

$\frac{d \mathrm{C} . \mathrm{V} .^{2}(X)}{d X}<0, X \geq X_{0} \quad$ we have: $\lim _{X \rightarrow \infty} \mathrm{C} . \mathrm{V}^{2}(X)=0$

This is the most "safe" situation.
c.
$M_{1}^{\prime}(X) \geq 0$
$M_{1}^{\prime \prime}(X) \geq 0$,
$X \geq X_{0}$ 
Again if $\mu(x)$ or even $\frac{d^{2} \log M_{1}(X)}{d X^{2}}$ is an increasing function the inequality:

$$
2 \exp \left[M_{1}^{\prime}(X)\right]-\mathrm{I} \leq \mathrm{C} . .^{2}(X)
$$

holds true. However, as there is the condition $\left|\frac{d M_{1}(X)}{d X}\right| \leq \mathrm{I}$, this situation implies that we can not have $M_{1}^{\prime \prime}(X)>0$ from a certain $X_{0}$ onward.

d.

$$
M_{1}^{\prime}(X) \geq 0, \quad M_{1}^{\prime \prime}(X) \leq 0, \quad X \geq X_{0}
$$

This case implies:

$$
\mu^{\prime}(X) \leq 0
$$

and we have:

$$
\mathrm{I} \leq \mathrm{C} . \mathrm{V} \cdot{ }^{2}(X) \leq 2 \exp \left[\int_{x}^{\bar{x}}\left(\mu(\xi)-\frac{\mathrm{I}}{M_{1}(\xi)}\right) d \xi\right]-\mathrm{I}
$$

Now we cannot make use of (4.4) in determining a bound for $\bar{x}$. However if we know that $M_{1}(X)$ has an upper bound $M$ we obtain at any rate the inequality:

$$
\mathrm{I} \leq \mathrm{C} \cdot \mathrm{V} \cdot{ }^{2}(X) \leq 2 \frac{M}{M_{1}(X)}-\mathrm{I}
$$

\section{A Different APPROACH}

The foregoing implies an application of Jensen's inequality to special expressions for: C.V. ${ }^{2}(X)$.

It is possible to obtain other, and for some important situations even better estimates for C.V. ${ }^{2}(X)$ by studying the formal solution for the differential equation of C.V. ${ }^{2}(X),(3,2)$ more closely. This solution is given by:

C.V. ${ }^{2}(X)=\left(\mathrm{I}+M_{1}^{\prime}(\bar{x})\right) \int_{x}^{\infty} \frac{\mathrm{I}}{M_{1}(x)} \exp \left[-\int_{x}^{x} \frac{\mathrm{I}}{M_{1}(\xi)}\left(\mathrm{I}-M_{1}^{\prime}(\xi)\right) d \xi\right] d x$

with $\bar{x}>X$.

$$
=\left(\mathrm{I}+M_{1}^{\prime}(\bar{x})\right) \frac{\mathrm{I}}{M_{1}(X)} \int_{x}^{\infty} \exp \left[-\int_{x}^{x} \frac{\mathrm{I}}{M_{1}(\xi)} d \xi\right] d x
$$


From (3.5) and remembering that:

$$
M_{1}(X)=\int_{x}^{\infty} \exp \left[-\int_{x}^{x} \mu(\xi) d \xi\right] d x,
$$

it follows that we can write:

$$
\text { C.V. }{ }^{2}(X)=\left(\mathrm{I}+M^{\prime}(\bar{x})\right) \frac{1}{2} \cdot\left[\mathrm{C} . \mathrm{V}^{2}(X)+\mathrm{I}\right]
$$

Solving for C.V. ${ }^{2}(X)$ we obtain the very simple formula:

$$
\text { C.V. }{ }^{2}(X)=\frac{\mathrm{I}+M_{1}^{\prime}(\bar{x})}{\mathrm{I}-M_{1}^{\prime}(\bar{x})} \text { with } \bar{x}>X
$$

Now we can set up the scheme:

\begin{tabular}{c|c|cc}
\hline$M_{1}^{\prime}(X)$ & $M_{1}^{\prime \prime}(X)$ & $\mathrm{C} \cdot \mathrm{V}^{2}(X)$ \\
\hline$\leq 0$ & $\geq 0$ & $\frac{\mathrm{I}+M_{1}^{\prime}(X)}{\mathrm{I}-M_{1}^{\prime}(X)} \leq \mathrm{C} \cdot \mathrm{V} \cdot{ }^{2}(X) \leq \mathrm{I}$ \\
$\leq 0$ & $\leq 0$ & 0 & $\leq \mathrm{C} \cdot V^{2} \cdot(X) \leq \frac{\mathrm{I}+M_{1}^{\prime}(X)}{\mathrm{I}-M_{1}^{\prime}(X)}$ \\
$\geq 0$ & $\geq 0$ & $\frac{\mathrm{I}+M_{1}^{\prime}(X)}{\mathrm{I}-M_{1}^{\prime}(X)} \leq \mathrm{C} \cdot \mathrm{V}^{2}(X)$ \\
$\geq 0$ & $\leq 0$ & $\mathrm{I}$ & $\leq \mathrm{C} \cdot V^{2} \cdot{ }^{2}(X) \leq \frac{\mathrm{I}+M_{1}^{\prime}(X)}{\mathrm{I}-M_{1}^{\prime}(X)}$ \\
\hline
\end{tabular}

In case there is any knowledge as regards the limit value of $\frac{\mathrm{I}+M_{1}^{\prime}(X)}{\mathrm{I}-M_{1}^{\prime}(X)}$ for $X \rightarrow \infty$ we have in addition:

$$
\lim _{x \rightarrow \infty} \mathrm{C} . V^{2}(X)=\lim _{X \rightarrow \infty} \frac{\mathrm{I}+M_{1}^{\prime}(X)}{\mathrm{I}-M_{1}^{\prime}(X)}
$$

October I966. 